\title{
In vitro Evaluation of the Antibacterial Potential of Niosome- Encapsulated Olive Leaf Extract
}

\author{
Aygul Can \\ Food Engineering Department, Engineering Faculty, \\ Canakkale Onsekiz Mart University, 17100, Canakkale, Turkey \\ E-mail: aygul.can@hotmail.com \\ Asli Kiraz \\ Medical Microbiology Department, Medical Faculty, \\ Canakkale Onsekiz Mart University, 17100, Canakkale, Turkey \\ E-mail: akiraz@comu.edu.tr \\ Cigdem Uysal Pala (Corresponding author) \\ Food Engineering Department, Engineering Faculty, \\ Canakkale Onsekiz Mart University, 17100, Canakkale, Turkey \\ E-mail: cigdemuysal@comu.edu.tr
}

The research is financed by Canakkale Onsekiz Mart University (BAP Project \# FLY-2016-958)

\begin{abstract}
Olive leaf extract (OLE) loaded niosomes as an antibacterial agent delivery system against Escherichia coli (ATCC 25922), Salmonella Typhimurium (ATCC 14028), Listeria innocua (ATCC 33090) and Methicillin-resistant Staphylococcus aureus (MRSA) were investigated by comparing with the antibacterial activity of free OLE. Niosomes were prepared using a lipid film hydration method followed by ultrasound-assisted size reduction technique and characterized in terms of morphology, entrapment efficiency $(41.92 \pm 0.81 \%)$, average particle size $(2.66 \pm 1.25 \mu \mathrm{m})$ and zeta-potential $(-34.16 \pm 6.48 \mathrm{mV})$. The minimum inhibitory concentrations (MIC) of free OLE and OLE loaded niosomes against strains were determined. The most susceptible strain against free OLE was the MRSA with MIC value of 2.34 $\mathrm{mg} / \mathrm{mL}$ OLE. However, the MIC value of OLE loaded niosomes against MRSA was $100 \mathrm{mg} / \mathrm{mL}$. Unfortunately, OLE loaded niosomes $(150 \mathrm{mg})$ with max capacity did not show any antibacterial effect against other three strains due to entrapment efficiency. In conclusion, OLE loaded niosomes has promising value as a novel antimicrobial delivery system to control MRSA.
\end{abstract}

Keywords: Olive leaf extract, microencapsulation, noisome, oleuropein, antibacterial activity.

DOI: $10.7176 / \mathrm{JSTR} / 5-6-01$

\begin{abstract}
1.Introduction
Recent advances in nanotechnology ensured that using lipid-based encapsulation technologies including liposomes and niosomes, for encapsulation of bioactive compounds has overgrown (Shishir et al. 2018). Biocompatible and degradable niosome developed as an alternative to the liposome is self-assembled vesicles in the presence of non-ionic surfactants and cholesterol (Khan and Irchhaiya 2016). Niosomes and liposomes are nearly the same from the point of their physical properties and formulations. Niosomes have some advantages such as higher chemical stability, adaptability of industrial production at low cost, ease of storage and sterilization to compare with liposomes (Aditya et al. 2017). It has been reported that using the niosome for encapsulating active compounds can improve the antimicrobial (Patel et al. 2015) and antioxidant (Liang et al. 2016) activities and the stability (Ruksiriwanich et al. 2014) of the compounds. Liposome-like technology can also mask the unpleasant taste of the therapeutic agent (Sajal et al. 2015). On the other hand, the use of suitable microencapsulation techniques for many purposes, such as increasing the stability of natural antimicrobials, masking bitter tastes, improving the diffusion properties of antimicrobials to the foods or pathogenic bacteria cells, are also among favorite research topics.
\end{abstract}

The olive tree (Olea europaea L.) is recognized for its economic importance in the Mediterranean 
countries. Rich phenolic content in their fruits and leaves with broad distribution exists (Silva et al. 2006). Because of interest to the biofunctionality of the phenolic compounds, the olive fruit and other parts of the olive tree have been investigated. Oleuropein is one of the major phenolic compounds that have antimicrobial activity in olive leaves (Esti et al. 1998). Plant-based antimicrobial compounds rise to prominence in the food industry with their therapeutic effect, and they can be utilized for food preservation and various pathogen-induced diseases (Tiwari et al. 2009). On the other hand, foodborne pathogens such as Escherichia coli, Salmonella spp., Campylobacter spp., Listeria monocytogenes, and Staphylococcus aureus are reported as a significant public health concern (Boskovic et al. 2015). The overuse of antibiotics against pathogen causing infections has made common the form of antibioticresistant strains, like methicillin-resistant Staphylococcus aureus (MRSA). MRSA threat human health in hospital and community, and cause of severe healthcare-associated infections, furthermore community-associated MRSA, livestock-associated MRSA, and healthcare-associated MRSA can be found in foods. Farms, livestock and people working in animal husbandry can be a significant source for the contamination of MRSA to foods, especially animal-based foods (Sergelidis and Angelidis 2017).

The major purpose of this study is to microencapsulate OLE using niosomal encapsulation technique and to determine the antibacterial activities of OLE and OLE loaded niosomes against food-borne pathogens including Listeria innocua (ATCC 33090), Escherichia coli (ATCC 25922), Salmonella Typhimurium (ATCC 14028) and Methicillin-resistant Staphylococcus aureus (MRSA).

\section{Materials and Methods}

2.1. Plant Material and Preparation of the Samples: Homogeneous powder from fresh leaves of Peranzana olive cultivar was prepared following the method suggested by Can $e t$ al. (2018). Powdered leaves were mixed with $80 \%$ aqueous ethanol (1:30 w:v) and exposed to an extraction process using a water bath (Memmert WNB 10 , Germany) at $80^{\circ} \mathrm{C}$ for 10 minutes. The ethanolic phase of the mixture was evaporated using a rotary evaporator (Büchi Rotavapor R-300, Switzerland) and lyophilized (Christ, Germany). The lyophilized sample was mixed with distilled water (1:7) (w/v) and centrifuged at 14000 rpm for 5 minutes (Centurion Scientific, UK). The supernatant was filtered through a $0.20 \mu \mathrm{m}$ PTFE filter and was the lyophilized second time. Samples were kept in at $-22{ }^{\circ} \mathrm{C}$ until the subsequent analyses were acquired.

2.2. Oleuropein Analysis: Oleuropein content of leaves was determined by using the methodology previously described by Al-Rimawi (2014). Oleuropein analysis was performed using an analytical HPLC system (Shimadzu LC 20A, Japan), equipped with a DAD (diode array detector) and an Inertsil C18 ODS 3 ( $250 \times 4.6 \mathrm{~mm}, 5 \mu \mathrm{m}$ particle size) reverse phase column. Oleuropein content was expressed as "mg oleuropein/g DW (dry weight) leaves."

2.3.Total Phenolic Content (TPC):Total phenolics in the samples were analyzed following the FolinCiocalteu method (Singleton and Rossi 1965) and expressed as mg GAE (gallic acid equivalents)/g DW leaves.

2.4. Preparation of Vesicles: Preparation of niosomes were done using the lipid film hydration method followed by ultrasound-assisted size reduction technique with minor modifications reported by Ruckmani and Sankar (2010). $100 \mathrm{mg} / \mathrm{mL}$ OLE in PBS (pH 7.4) was prepared under sterile condition. OLE was filtered through a $0.20 \mu \mathrm{m}$ PTFE filter and stored at $4^{\circ} \mathrm{C} .500 \mathrm{mg}$ Span 60 and $500 \mathrm{mg}$ cholesterol were dissolved in $8 \mathrm{~mL}$ chloroform, and the organic solvent was removed under vacuum at $150 \mathrm{rpm}, 60^{\circ} \mathrm{C}$ for 2 hours using the rotary evaporator to form the thin film. The dried film hydrated with $5 \mathrm{~mL}$ OLE $(100 \mathrm{mg} / \mathrm{mL})$ prepared before, followed by shaking at $150 \mathrm{rpm}, 60^{\circ} \mathrm{C}$ for 2 hours using shaking incubator (Biochemical Incubator SPX-70S, China) and ultrasonic bath (Isolab, Germany) at $60^{\circ} \mathrm{C}$ for 20 minutes. For the preparation of empty niosomes, the dried film was hydrated with sterile PBS (pH 7.4) without OLE with same hydration procedure. The niosomal suspension was maturated night long at $4{ }^{\circ} \mathrm{C}$. The maturated niosomes were sonicated at $30^{\circ} \mathrm{C}$ for $30 \mathrm{~min}$ by ultrasonic bath sonicator to achieve a niosomal dispersion containing both free and entrapped OLEs of varying size and stored at $4^{\circ} \mathrm{C}$ for further analysis.

\subsection{Characterization of Niosomes}

2.5.1. Entrapment Efficiency: The unentrapped OLE was separated from the niosomal dispersion by centrifugation method with modifications reported by Pawar et al. (2016). OLE loaded niosomes were washed with PBS and dispersion was centrifuged at $4^{\circ} \mathrm{C}, 15000 \mathrm{rpm}$ for $30 \mathrm{~min}$ using a refrigerated centrifuge (Hettich Universal 320R, Germany). The concentration of the free OLE in the supernatant was

2| P a g e

www.iiste.org 
analyzed by the Folin-Ciocalteu method. The percentage of OLE entrapment in niosomes was calculated as:

Entrapment efficiency $(\% \mathrm{EE})=[(C t-C f) / C t] \times 100$

Where $C t$ is the total and $C f$ is the free OLE concentration

2.5.2. Vesicle Diameter: The niosomal dispersion was prepared at $0.5 \mathrm{mg} / \mathrm{mL}$ concentration with 0.01 M potassium chloride for use in the dynamic light scattering (DLS) method. A Particle Size Analyzer (90 Plus, Brookhaven Instrument Corp., USA) was used to determine the average particle sizes of niosomes.

2.5.3. Zeta Potential: The niosomal dispersion was prepared at $0.25 \mathrm{mg} / \mathrm{mL}$ concentration with $0.01 \mathrm{M}$ potassium chloride. Zeta potential measurement of niosomal dispersion was performed using the ZetaPals Zeta Potential Analyzer BIC (Brookhaven Inst. Corp., USA).

2.5.4. Optical Microscopy: The niosomal dispersion was viewed under an optical microscope (Zeiss, PrimoVert, Germany) to observe the shape and lamellar nature of the vesicle before and after sizing.

2.5.5. Transmission Electron Microscopy (TEM): A drop of niosomal dispersion was placed onto a carbon-coated grid and allowed to air dry to a thin film. The effective formation of the vesicles was investigated at $120 \mathrm{kV}$ using TEM (JEOL JEM 1400 Plus TEM, Japan).

2.5.6. Scanning Electron Microscopy (SEM): A drop of niosomal dispersion was placed on clear glass stub and air dried. The sample was coated with Palladium-Gold. Surface morphology of samples was viewed at $10 \mathrm{kV}$ using SEM (FE-SEM JFM 7100F EDS, JEOL, Japan).

2.6. Minimum Inhibitory Concentration (MIC) and Minimum Bactericidal Concentration (MBC): The MICs of free and OLE loaded niosomes were determined against four bacteria using standardized broth microdilution technique (Kuete and Metuno 2007). $750 \mathrm{mg} / \mathrm{mL}$ OLE in PBS (pH 7.4) was prepared under sterile condition. $750 \mathrm{mg} / \mathrm{mL}$ stock OLE solution was diluted with PBS to prepare concentrations (750-37.5 mg/mL) against L. innocua, E. coli and $S$. Typhimurium, and concentrations $(75-0.29 \mathrm{mg} / \mathrm{mL})$ against MRSA. $300 \mathrm{mg} / \mathrm{mL}$ stock niosomal dispersion was diluted with PBS to prepare seven concentrations (300-6.25 mg/mL) against L. innocua, E. coli and $S$. Typhimurium, six concentrations (200-6.25 mg/mL) against MRSA. $100 \mu \mathrm{L}$ of each sample solution (OLE or niosomes) was added to the sterile 96 wells that contained $95 \mu \mathrm{L}$ Mueller-Hinton Broth and $5 \mu \mathrm{L}$ bacterial suspensions $\left(10^{7}\right.$ $\mathrm{CFU} / \mathrm{mL}$ ). The final inoculum concentration in the well is $10^{5} \mathrm{CFU} / \mathrm{mL}$. In order to control the growth of the microorganism, $195 \mu \mathrm{L}$ MHB and $5 \mu \mathrm{L}$ of inoculum $\left(10^{7} \mathrm{CFU} / \mathrm{mL}\right)$ were pipetted into well. For sterility control, $200 \mu \mathrm{L}$ MHB was incubated. Bacteria were incubated in a spectrophotometer at $37^{\circ} \mathrm{C}$ for 18 hours and absorbance was measured at $620 \mathrm{~nm}$ per hour. Experiments were performed in duplicate. The MBC value was higher than the MIC value and indicated that there was no colony growth from samples incubated at $37^{\circ} \mathrm{C}$ for $18 \mathrm{~h}$.

\subsection{Statistical analyses}

Statistical Analysis Systems Version 9 (SAS Institute Inc, Cary, NC, USA) with MiXED procedure was used to analyze the data.

\section{Result and Discussion}

\subsection{Characterization of OLE Loaded Niosomes}

The ratio of surfactant, cholesterol and other additives are important parameters as well as the ratio of the active substances to the total lipids added to the formulation (Mokhtar et al. 2008). Span 60: Cholesterol $(1: 1 \mathrm{w} / \mathrm{w})$ ratio was selected since it gives the optimal encapsulation efficiency according to many studies (Balakrishnan et al. 2009, Shilakari et al. 2016). It has been reported that Span 60 (HLB 4.7) enables higher encapsulation efficiency compared to Span 20, due to its high phase transition temperature. On the other hand, the permeability of biomolecular the membranes and structure of the hydrocarbon chain in surfactant are principal for encapsulation of hydrophilic molecules in lipid-based capsules. Span 60 has saturated acyl chains in the semi-solid gel state under normal ambient conditions, so leakage of the hydrophilic active substance from the vesicles prepared with span 60 is less (Abdelkader et al. 2010). Cholesterol has a significant influence on membrane properties. Nematollahi et al. (2017) reported that while membrane stability increase with cholesterol usage in the niosomal formulation, membrane fluidity decrease.

3| P a g e www.iiste.org 
Characterization of the prepared niosomes was achieved by determining encapsulation efficiency, vesicle diameter, and zeta potential. Light microscopy, SEM, and TEM were also used to examine the morphology of niosomes. Also, the encapsulation efficiency of the niosomes is the most essential parameter in terms of pharmacy with its potential use in drug delivery systems (Balakrishnan et al. 2009). The encapsulation efficiency of the OLE loaded niosomes was $41.92 \pm 0.81 \%$ after free OLE was removed from the capsules. Particle size which varies with the HLB value and the chain length of the surfactant is an essential factor for encapsulation efficiency and release of the active substance (Uchegbu and Vyas 1998). The ultrasonic method $\left(30^{\circ} \mathrm{C}, 30 \mathrm{~min}\right)$ was applied in order to reduce the size of niosomes, and the average vesicle diameter of the prepared OLE loaded, and empty niosomes were $2.66 \pm 1.25 \mu \mathrm{m}$ and $2.37 \pm 1.41 \mu \mathrm{m}$, respectively.

Similarly, the diameter of niosomes prepared by Aswathy and John (2014) was reported between 2.58 $\mu \mathrm{m}$ and $3.13 \mu \mathrm{m}$. The zeta potentials of OLE loaded, and empty niosomes were measured as $-34.16 \pm$ $6.48 \mathrm{mV}$ and $-70.68 \pm 4.75 \mathrm{mV}$, respectively. The charge on the surface of the vesicles produces a homogeneous dispersion because of producing a repulsive force between the vesicles. Values of zeta potential above $30 \mathrm{mV}$ (positive or negative) are adequately high for electrostatic stability and a stable dispersion (Mahajan et al. 2012). Patel et al. (2015) reported that the zeta potentials of propolis-loaded and free niosomes were $-33.2 \mathrm{mV}$ and $-38.8 \mathrm{mV}$, respectively.

As shown in Figure 1, niosomes are seen in different shapes and sizes after maturation. Vesicle sizes prominently decreased, and the distribution of vesicles became more homogenous after sizing with the ultrasound application. SEM micrographs reveal the empty and OLE loaded niosomes are spherical and TEM micrographs confirm the presence of homogeneously dispersed vesicles. However, while the shapes of the empty niosomes are generally spherical, different forms were also observed in OLE loaded niosomes.

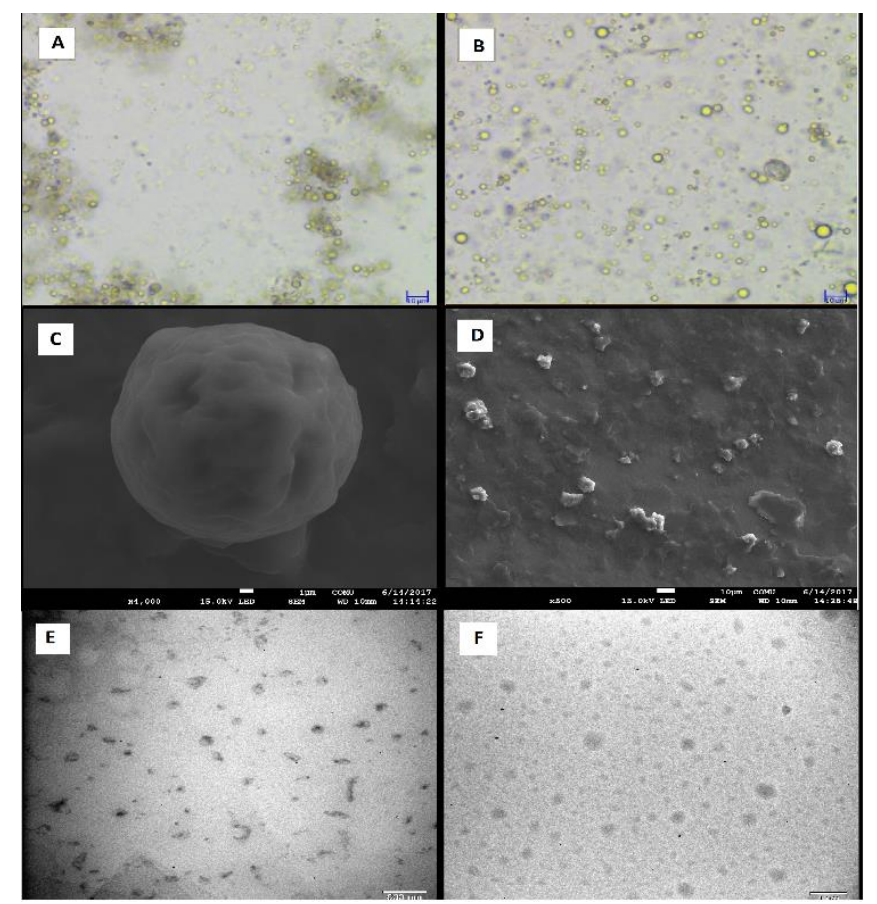

Figure 1. (A) OLE loaded niosome after maturation (x10), (B) OLE loaded niosome after sizing (x10), (C) SEM micrograph of OLE loaded niosomes (4000x), (D) SEM micrograph of OLE loaded niosomes (x4000) (E) TEM micrograph of OLE loaded niosomes (x500) (F) TEM micrograph of empty niosomes.

\subsection{In Vitro Antibacterial Activity of free OLE and OLE Loaded Niosomes}

The MIC and MBC values of free OLE and OLE loaded niosomes for each test strain are given in Table 1. MICs of free OLE were $2.34 \mathrm{mg} / \mathrm{mL}$ (including $70.60 \pm 3.68 \mu \mathrm{g}$ oleuropein) against MRSA and 150 $\mathrm{mg} / \mathrm{mL}$ (including $3380.00 \pm 169.71 \mu \mathrm{g}$ oleuropein) against E. coli, S. Typhimurium and L.innocua. The growths of bacteria have slowed but not stopped at OLE concentrations lower than $2.34 \mathrm{mg} / \mathrm{mL}$ for MRSA and lower than $150 \mathrm{mg} / \mathrm{mL}$ for $E$. coli and $S$. Typhimurium, compared to inoculum growth (Figure 2). The antimicrobial activity of free OLE depends on some parameters such as leaf origin, harvesting time, climate, extract preparation methods (Ranalli et al. 2006) and also oleuropein content of extract as 
we observe in this study. The antibacterial activity of oleuropein results from the presence of the orthodiphenolic system due to the number and location of hydroxyl groups of the phenolics (Cowan 1999). Pereira et al.(2007) reported that OLE damages the cell membrane permeability due to the protein denaturation. Moreover, isolated phenolic compounds can be less active than plant extracts because of the interaction of phenolics each other.

Table 1. Viable counts in samples of OLE and OLE loaded niosomes inoculated with listed strains incubated at $37^{\circ} \mathrm{C}$ for 18 hours

\begin{tabular}{|c|c|c|c|c|c|c|c|}
\hline Inoculum & Sample & $\begin{array}{l}\text { Conc. } \\
\text { (mg/mL) }\end{array}$ & $\begin{array}{l}\text { Oleuropein } \\
\text { content } \\
(\mu \mathrm{g} / \mathrm{mL})\end{array}$ & $\begin{array}{c}\text { tinitial } \\
\left(\log _{10} C F U / m L\right)\end{array}$ & $\begin{array}{c}\mathrm{t}_{18 \mathrm{~h}} \text { (inoculumcontrol) } \\
\left(\log _{10}\right. \\
\text { CFU } / \mathrm{mL})\end{array}$ & $\begin{array}{c}\mathrm{t}_{18 \mathrm{~h}} \\
\left(\log _{10}\right. \\
\text { CFU/mL) }\end{array}$ & $\begin{array}{c}\text { Difference }^{* *} \\
\left(\log _{10}\right. \\
\text { CFU/mL) }\end{array}$ \\
\hline \multirow{7}{*}{ MRSA } & OLE & 2,34 & $70,60 \pm 3,68$ & $6,02 \pm 0,03^{*}$ & $9,17 \pm 0,09$ & $\leq 1$ & $-9,17$ \\
\hline & \multirow{3}{*}{ Niosome } & 50 & $76,53 \pm 2,79$ & $5,89 \pm 0,05$ & $9,17 \pm 0,09$ & $8,75 \pm 0,10$ & $-0,42$ \\
\hline & & 100 & $153,10 \pm 7,41$ & $5,87 \pm 0,05$ & $9,17 \pm 0,09$ & $5,57 \pm 0,09$ & $-3,60$ \\
\hline & & 150 & $229,60 \pm 3,61$ & $5,99 \pm 0,01$ & $9,17 \pm 0,09$ & $\leq 1$ & $-9,17$ \\
\hline & \multirow{3}{*}{$\begin{array}{l}\text { Empty } \\
\text { Niosome }\end{array}$} & 50 & - & $6,09 \pm 0,08$ & $9,17 \pm 0,09$ & $8,91 \pm 0,07$ & $-0,26$ \\
\hline & & 100 & - & $5,93 \pm 0,03$ & $9,17 \pm 0,09$ & $8,98 \pm 0,07$ & $-0,19$ \\
\hline & & 150 & - & $5,93 \pm 0,03$ & $9,17 \pm 0,09$ & $9,15 \pm 0,11$ & $-0,02$ \\
\hline \multirow{3}{*}{ E. coli } & OLE & 150 & $3380 \pm 169,71$ & $5,37 \pm 0,07$ & $9,01 \pm 0,15$ & $\leq 1$ & $-9,01$ \\
\hline & Niosome & 150 & $229,60 \pm 3,61$ & $5,65 \pm 0,05$ & $9,01 \pm 0,15$ & $9,02 \pm 0,09$ & 0,01 \\
\hline & Empty Niosome & 150 & - & $5,61 \pm 0,02$ & $9,01 \pm 0,15$ & $9,03 \pm 0,11$ & 0,02 \\
\hline \multirow{3}{*}{ S. Typhimurium } & OLE & 150 & $3380 \pm 169,71$ & $5,81 \pm 0,08$ & $9,24 \pm 0,05$ & $\leq 1$ & $-9,24$ \\
\hline & Niosome & 150 & $229,60 \pm 3,61$ & $6,24 \pm 0,01$ & $9,24 \pm 0,05$ & $9,19 \pm 0,06$ & $-0,05$ \\
\hline & Empty Niosome & 150 & - & $6,49 \pm 0,07$ & $9,24 \pm 0,05$ & $9,28 \pm 0,06$ & 0,04 \\
\hline \multirow{3}{*}{ L. innocua } & OLE & 150 & $3380 \pm 169,71$ & $6,50 \pm 0,06$ & $7,65 \pm 0,07$ & $\leq 1$ & $-7,65$ \\
\hline & Niosome & 150 & $229,60 \pm 3,61$ & $6,49 \pm 0,14$ & $7,65 \pm 0,07$ & $8,23 \pm 0,08$ & 0,58 \\
\hline & EmptyNiosome & 150 & - & $6,64 \pm 0,13$ & $7,65 \pm 0,07$ & $7,21 \pm 0,24$ & $-0,44$ \\
\hline
\end{tabular}

*Values are expressed as means \pm S.D. $(n=2)$, Detection limit $\leq 1 \log _{10} \mathrm{CFU} / \mathrm{mL}$

${ }^{* *}$ Difference $=\mathrm{t}_{18 \mathrm{~h}}$ (inoculum control) $-\mathrm{t}_{18 \mathrm{~h}}$

The MIC values of OLE loaded capsules against E. coli, S. Typhimurium and L. innocua were lower than $150 \mathrm{mg} / \mathrm{mL}$, as seen in Figure 3. However, according to the results of the MBC for the verification of the microdilution method, no inhibition was observed for these pathogens at $150 \mathrm{mg} / \mathrm{mL}$ of OLE loaded niosomes. Therefore, deviations in the graphs obtained by broth microdilution method may be the milky color of highly concentrated niosomes analyzed spectrophotometrically.

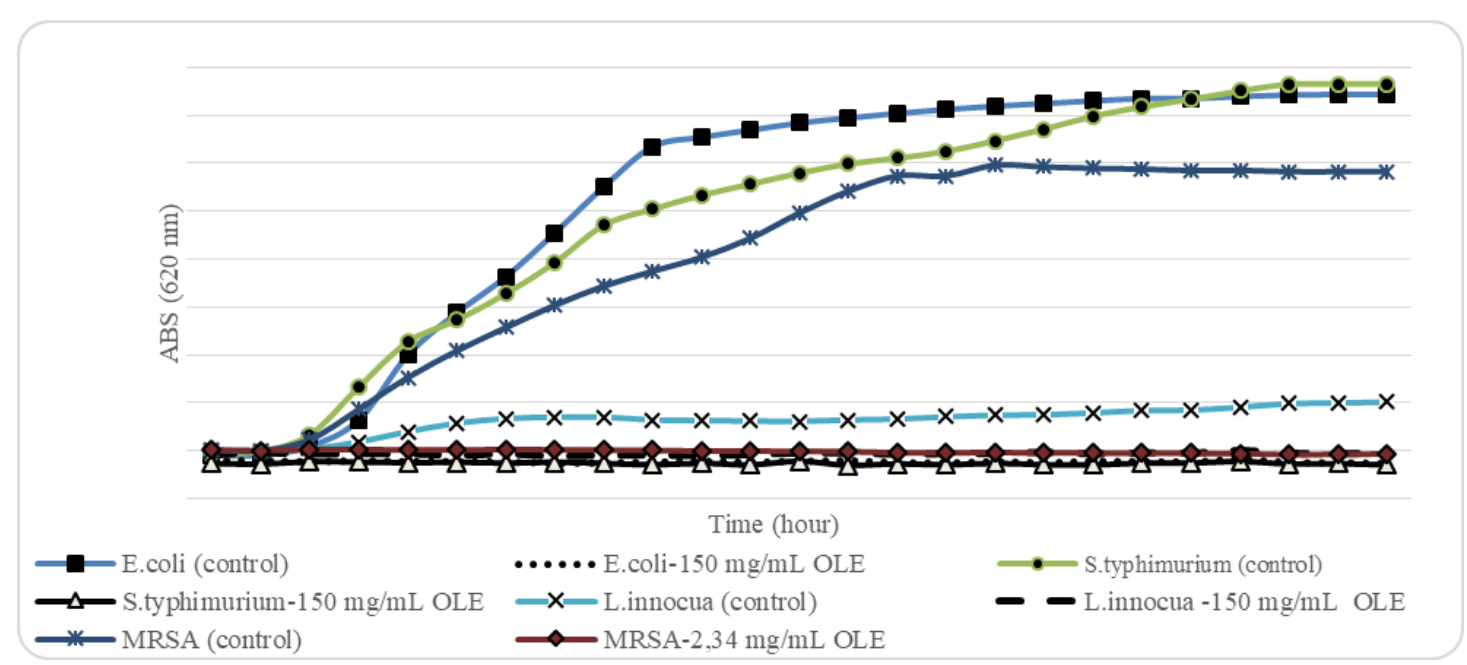

Figure 2. OLE concentrations against E. coli, S. Typhimurium, L. innocua and MRSA. 


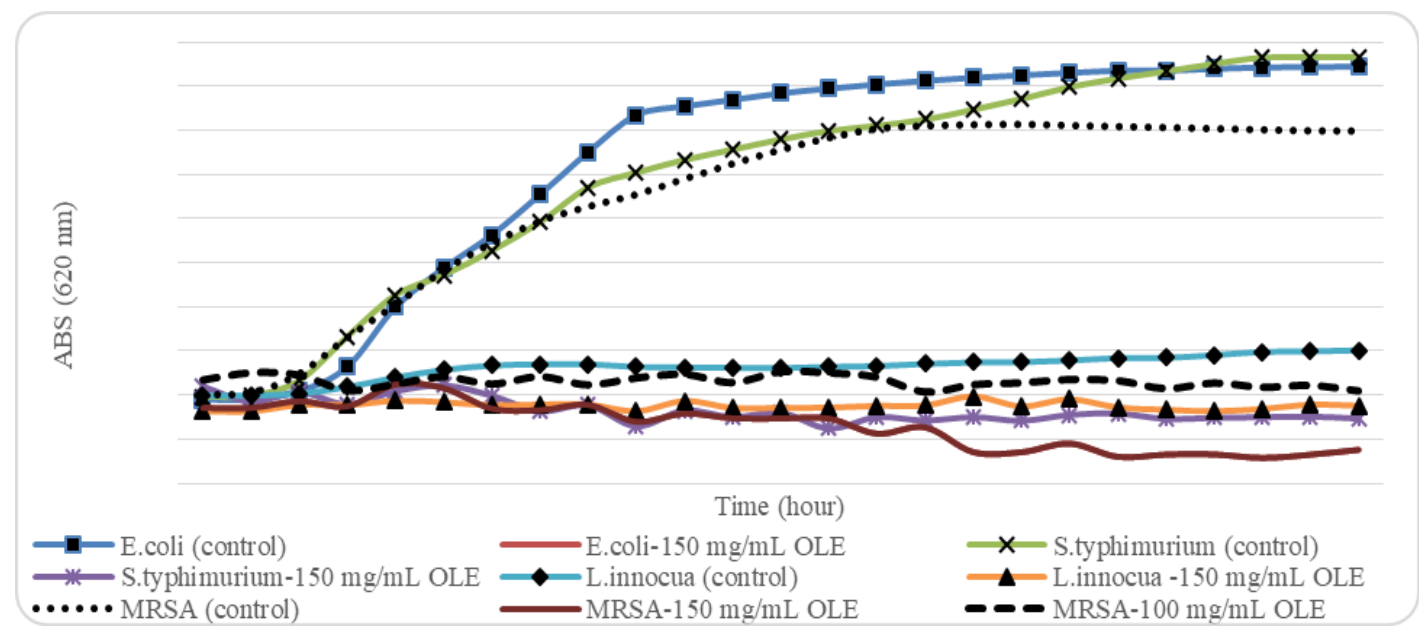

Figure 3. OLE loaded niosome concentrations against E. coli, S. Typhimurium, L. innocua and MRSA.

For post-incubation samples ( $\mathrm{t}_{18}$, Table 1$)$, neither the difference between the count of $E$. coli treated with OLE loaded niosomes and count of control inoculum is not significant $(\mathrm{P}>0.05)$ nor the difference between S.Typhimurium treated with OLE loaded niosomes and control. Moreover, OLE loaded niosomes $(150 \mathrm{mg} / \mathrm{mL})$ increased the growth of L. innocua by $0.58 \log (\mathrm{P}<0.005)$. The encapsulated OLE is not sufficient for the inhibition of E. coli, S.Typhimurium and L. innocua. Also, empty capsules do not show any antibacterial effect against strains (Table 1). On the other hand, the growth of MRSA was not observed at $150 \mathrm{mg}$ (with $229.60 \pm 3.61 \mu \mathrm{g}$ oleuropein)/mL of OLE loaded capsule treatment $(\mathrm{P}<0.0001)$. The MIC and MBC of OLE loaded niosomes against MRSA were $100 \mathrm{mg}$ (containing $153.10 \pm 7.41 \mu \mathrm{g}$ oleuropein)/ml and $150 \mathrm{mg}$ (containing 229.60 $\pm 3.61 \mu \mathrm{g}$ oleuropein)/mL respectively. OLE-loaded niosomes show potential as an antimicrobial agent only against MRSA among pathogens involved in this research.

OLE content of niosomes decreased because of low encapsulation efficiency (41.92 $\pm 0.81 \%)$. For this reason, the OLE loaded niosomes $(150 \mathrm{mg} / \mathrm{mL})$ used in the antibacterial activity assays against $E$. coli, $S$. Typhimurium and L. innocua, did not contain enough oleuropein for inhibition. Besides, when OLE and OLE loaded niosomes are compared quantitatively against MRSA, about 3.25 times more oleuropein-containing niosomes seem to be active on inhibition of MRSA when compared to oleuropein content. In literature, results of the agar diffusion method have shown that gatifloxacin loaded niosomes have similar antibacterial activity with aqueous gatifloxacin against Pseudomonas aeruginosa and S.aureus (Mahajan et al. 2012). In another study, MIC values of the free and niosomal moxifloxacin were found the same at $0.87 \mu \mathrm{g} / \mathrm{mL}$ against $S$.aureus, although MIC values of the free moxifloxacin and loaded niosome were found at $6.25 \mu \mathrm{g} / \mathrm{mL}$ and $3.125 \mu \mathrm{g} / \mathrm{mL}$ respectively against $P$. aeruginosa (Sohrabiet et al. 2016). Patel et al. (2015) prepared propolis-loaded niosomes and has reported that MIC values of propolis loaded noisome and ethanolic propolis solution against $S$. aureus was $0.3 \mathrm{mg} / \mathrm{mL}$ and $0.5 \mathrm{~g} / \mathrm{mL}$, respectively. The MIC values of free vancomycin and vancomycin-loaded niosome against MRSA were reported as $8 \mu \mathrm{g} / \mathrm{mL}$ and $1 \mu \mathrm{g} / \mathrm{mL}$, respectively (Barakat et al. 2014).

\section{Conclusion}

In recent years, using phenolic compounds has attracted considerable attention from the food and pharmaceutical industries because of their functional properties. In this study, in vitro use of OLE as an antibacterial agent has shown a significant potential when assessed against $L$. innocua ATCC 33090, E. coli ATCC 25922, S. Typhimurium ATCC 14028 and methicillin-resistant $S$. aureus (MRSA). MRSA has susceptible deficient concentrations of OLE that can reduce potential risks particularly in the field of health. On the other hand, niosomal form of OLE was developed to offer useful and promising antimicrobial agent carrier system in order to enhance the food shelf-life and safety applications. In vitro antibacterial potential of OLE loaded niosome can be improved by using new formulations and techniques. These results warrant further research on oleuropein purification from OLE to increase the loading efficiency of niosome formulations to improve antimicrobial efficacy. 


\section{Acknowledgements}

Oral presentation of this study performed at the Joint Science Congress of Materials and Polymers (ISCMP, 25-28 August 2017), Ohrid-Macedonia.

\section{Conflict of interest}

All of the authors declare that there is no conflict of interest.

\section{References}

Abdelkader, H., Kamal, A., and Alany, R.G., 2010. Preparation of niosomes as an ocular delivery system for naltrexone hydrochloride : Physicochemical characterization. Pharmazie, 65(11), 1-7.

Aditya, N.P., Espinosa, Y.G., and Norton, I.T., 2017. Encapsulation systems for the delivery of hydrophilic nutraceuticals: Food application. Biotechnology advances, 35(4), 450-457.

Al-Rimawi, F., 2014. Development and validation of a simple reversed-phase HPLC-UV method for determination of oleuropein in olive leaves. Journal of food and drug analysis, 22(3), 285-289.

Aswathy, K.S., and John, R.O., 2014. Design, characterization and evaluation of latanoprost niosomes for glaucoma treatment. Journal of scientific and innovative research, 3(6), 558-562.

Balakrishnan, P., et al., 2009. Formulation and in vitro assessment of minoxidil niosomes for enhanced skin delivery. International journal of pharmaceutics, 377(1-2), 1-8.

Barakat, H., et al., 2014. Vancomycin-eluting niosomes: a new approach to the inhibition of staphylococcal biofilm on abiotic surfaces. The american association of pharmaceutical, 15(5), 1263-1274.

Boskovic, M., et al., 2015. Antimicrobial activity of thyme (Tymus vulgaris) and oregano (Origanum vulgare) essential oils against some food-borne microorganisms. Procedia food science, 5, 18-21.

Can, A., et al., 2018. The potential of near and mid-infrared spectroscopy for rapid quantification of oleuropein, total phenolics, total flavonoids and antioxidant activity in olive tree (Olea europaea) leaves. Journal of food measurement and characterization, 12(4), 2747-2757.

Cowan, M.M., 1999. Plant products as antimicrobial agents. Clinical microbiology reviews, 12(4), 564-582.

Esti, M., Cinquanta, L., and La Notte E., 1998. Phenolic compounds in different olive varieties. Journal of agricultural and food chemistry, 46(1), 32-35.

Khan, R. and Irchhaiya, R., 2016. Niosomes: a potential tool for novel drug delivery. Journal of pharmaceutical investigation, 46, 195-204.

Kuete, V., and Metuno, R., 2007. Antimicrobial activity of the methanolic extracts and compounds from Treculia obovoidea (Moraceae). Journal of ethnopharmacology, 112(3), 531-536.

Liang, R., et al., 2016. Niosomes Consisting of tween-60 and cholesterol improve the chemical stability and antioxidant activity of (-)-epigallocatechin gallate under intestinal tract conditions. Journal of agricultural and food chemistry, 64(48), 9180-9188.

Mahajan, H.S., et al., 2012. Niosomes encapsulated with gatiflaxacin for ocular drug delivery. Recent advances in pharmaceutical science research, 11(11), 28-39.

Mokhtar, M., et al., 2008. Effect of some formulation parameters on flurbiprofen encapsulation and release rates of niosomes prepared from proniosomes. International journal of pharmaceutics, 361(1-2), 104-111. 
Nematollahi, M.H., et al., 2017. Changes in physical and chemical properties of niosome membrane induced by cholesterol: a promising approach for niosome bilayer intervention. RSC advances, 7(78), 49463-49472.

Patel, J., et al., 2015. Potentiating antimicrobial efficacy of propolis through niosomal-based system for administration. Integrative medicine research, 4(2), 94-101.

Pawar, H.A., Attarde, V.B., and Parag Subhash, G., 2016. Optimization of bifonazole-loaded nisomal formulation using plackett-burman design and $2^{3}$ Factorial Design. Open pharmaceutical sciences journal, 3(1), 31-48.

Pereira, A.P., et al., 2007. Phenolic compounds and antimicrobial activity of olive (Olea europaea L. Cv. Cobrançosa) leaves. Molecules, 12(5), 1153-1162.

Ranalli, A., et al., 2006. Factors affecting the contents of iridoid oleuropein in olive leaves (Olea europaea L.). Journal of agricultural and food chemistry, 54(2), 434-440.

Ruckmani, K., and Sankar, V., 2010. Formulation and Optimization of zidovudine niosomes. The american association of pharmaceutical scientists, 11(3), 1119-1127.

Ruksiriwanich, W., Sringarm, K., and Jantrawut, P., 2014. Stability enhancement of Celastrus paniculatus seed oil by loading in niosomes. Asian journal of pharmaceutical and clinical research, 7(2), 186-191.

Sajal, J.K., Uday, S.R., and Surendra, V., 2015. Taste masking in pharmaceuticals : an update. Journal of pharmacy research, 1, 126-130.

Sergelidis, D., and Angelidis, A.S., 2017. Methicillin-resistant Staphylococcus aureus: a controversial food-borne pathogen. Letters in applied microbiology, 64(6), 409-418.

Shilakari Asthana, G., Sharma, P.K., and Asthana, A., 2016. in vitro and in vivo evaluation of niosomal formulation for controlled delivery of clarithromycin. Scientifica, 2016.

Shishir, M.R.I., et al., 2018. Advances in micro and nano-encapsulation of bioactive compounds using biopolymer and lipid-based transporters. Trends in food science and technology, 78, 34-60.

Silva, S., et al., 2006. Phenolic compounds and antioxidant activity of Olea europaea L. fruits and leaves. Food science and technology international, 12(5), 385-395.

Singleton, V.L., and Rossi, J.A., 1965. Colorimetry of total phenolics with phosphomolybdicphosphotungstic acid reagents. American journal of enology and viticulture, 16, 144-158.

Sohrabi, S., et al., 2016. Chitosan gel-embedded moxifloxacin niosomes: An efficient antimicrobial hybrid system for burn infection. International journal of biological macromolecules, 85, 625633.

Tiwari, B.K.,et al.,2009. Application of natural antimicrobials for food preservation. Journal of agricultural and food chemistry, 57(14).

Uchegbu, I.F., and Vyas, S.P., 1998. Non-ionic surfactant based vesicles (niosomes) in drug delivery. International journal of pharmaceutics, 172(1-2), 33-70. 